\title{
Review of: "Bone-Targeted Erythrocyte-Cancer Hybrid Membrane-Camouflaged Nanoparticles For Enhancing Photothermal And Hypoxia-Activated Chemotherapy of Bone Invasion By OSCC"
}

\author{
Lin $\mathrm{Cai}^{1}$
}

1 Wuhan University

Potential competing interests: The author(s) declared that no potential competing interests exist.

Owing to the Asp8 and cell membrane, Asp8[H40-TPZ/IR780@(RBC-H)] NPs can achieve the precise bone and tumor targeting. The excellent antitumor effect of Asp8[H40-TPZ/IR780@(RBC-H)] NPs through photothermal and hypoxia-activated chemotherapy has been testified. Moreover, the bone invasion by OSCC can also been inhibited. this provided a good way for future treatment about the tumor which usually cause bone destruction. but the in vitro antitumor assay should be performed more logically and systematically.

$1 \square$ In figure $3 c$ and $d$, the subgroups were labeled as A, B and C, while the describe in footnote is i, ii and iii.

2 , the subgroups in figure $3 f$ should be consistent with figure $3 \mathrm{~g}$.

3, In the part of Results And Discussion, Anticancer assay in vitro, line 8, and in figure 3e, did the Asp8[H40-IR780@(RBC-H)] and Asp8[H40-TPZ/IR780@(RBC-H)] be misused ? At the part, line 21, the description in figure $3 f$, red fluorescence was observed in each group without laser irradiation? Or green fluorescence?

4, the data of cell viability of TPZ and IR780 with different concentration treated with or without irradiation should be performed.

5, In the description of Fig. 3e, how the author drawn the conclusion “These findings indicate that TPZ had no antitumor activities under a normoxic environment while it could be active in hypoxic environments by inducing the production of toxic oxidizing radicals". No data about the antitumor activities under a normoxic environment has been provide. And the part should be rewritten.

6, How to ensure the mouse model with invaded right mandibular be successfully establised ? by microCT 
? If have, the data should be provided.

7, Excellent anticancer effect of Asp8[H40-TPZ/IR780@(RBC-H)] without irradiation has been obtained in figure 3f, did the anticancer effect of Asp8[H40-TPZ/IR780@(RBC-H)] without irradiation in vivo have been testified? 